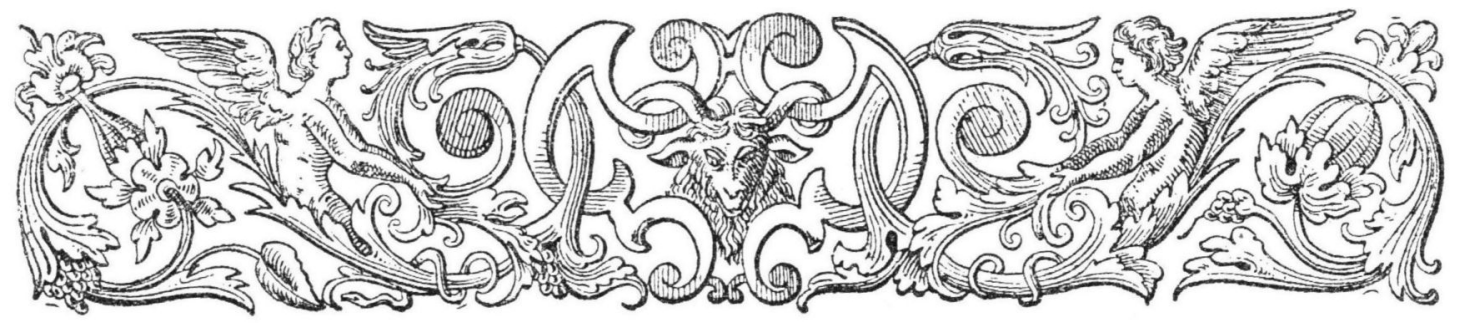

\title{
NIEDERLÄNDISCHE KUNST
}

IN DEN

\author{
GALERIEN MANSI ZU LUCCA \\ VON
}

EMIL JACOBSEN.

NTER den Collectionen niederländischer Kunst in Italien kommt gewiss der Galerie des Marchese Gerolamo MAnsi in Lucca, besonders durch ihren reichen Bestand holländischer Bilder, ein hervorragender Platz zu. Ich hege demnach die Hoffnung, dass nachfolgender kurzer Bericht über denselben den Lesern des "Oud-Holland" nicht unwillkommen sein dürfte. Marchese RafaEllo MaNSI, der Ur-urgrossvater des jetzigen Besitzers der Galerie, des Marchese Rafaello Orsetti Mansi, hat die Sammlung durch seine Heirath mit einer Parensi im J. I742 bekommen, und ein Ahne dieser PAREnsi soll im J. I676 mit einem Fräulein VAN DIEMEN aus Amsterdam verheirathet sein. So erklärt es sich, dass die Sammlung eine für Italien befremdend grosse Anzahl holländischer Bilder aufzuweisen hat.

Von der anderen kleineren Galerie MaNsI (in Piazza Maria Bianca) habe ich auch im Nachfolgenden einige Bilder erwähnt. Als ich dieselbe besuchte, war sie
\end{abstract}


NIEDERLÄNDISCHE KUNST IN DEN GALERIEN MANSI ZU LUCCA.

indessen wegen des Todes ihres Besitzers zur Versteigerung verurtheilt. Diese hat jedoch noch nicht stattgefunden.

\section{DiE GALERIE GEROLAMO MANSI.}

C. H. Verelst. Bildnisse. In der glatten Behandlung schon eine Vorahnung des ADriaAn van der WerfF. Beide signirt: C H Verelst F i6 66. $\mathrm{Ob}$ mit dem Sittenmaler PIETER Verelst verwandt?

David Vinckioons. Bauerngelag. In lackrother Farbe strahlend.

Jan Martsen De Jonge. Ein Bauer im Kampfe mit Marodeuren. Das hochhängende Bild ist signirt und mit der Jahreszahl I639 versehen.

Jan WeEnix. Schöner Park mit Fontaine: im Vordergrunde eine reich ornamentirte Vase, ein tother Papagei und der unvermeidliche todte Hase. Alles malerisch geordnet. $\mathrm{Zu}$ seinen besseren Bildern gehörend.

Pieter Claesz. Das Früstück. Breit und pastos gemalt. Signirt: PC I653.

Esaias VAN DE VELDE. Zwei kleine Landschaften, voll bezeichnet, Gelbgrünlicher Ton. Mit Strichen und Pricken mehr gezeichnet als gemalt. Flüchtig ausgeführte Skizzen des Meisters.

Stilleben: eine Schale mit Trauben und Pfrsichen. Feiner, olivenartiger Ton, leuchtende Farben. Mit einer sehr schwer zu entziffernden Signatur aus den Buchstaben DL T Z E T R (?), theilweise ineinander geschlungen.

Mattinas Withoos. Ein grosses, flottgemaites, prächtiges Gartenbild (die Villa d'Este). Mit Architektur, Bildsäulen, antiken Vasen belebt und voll rother prangender Blumen. Etwas in der Art des JAN WEEnIx und doch verschieden behandelt. Das Bild ist signirt und trägt die Jahreszahl i 666.

Pieter Paulus Rubens zugeschrieben. Die zwei Bilder mit Satyren und Nymphen sind wohl tüchtige Arbeiten aus der Schule des RUBENS, jedoch nicht von dem Meister selbst.

Der segnende Heiland. Halbfigur. In einem tothen goldverbrämten Mantel eingehüllt. Die rechte Hand segnend erhoben, die andre auf die mit einem Kreuz gekrönte Weltkugel gestützt. Steht Halbfiguren Christi von QUENTIN Matsys nahe. 
Rombout van Troyen. Der Brand Trojas. Nicht ohne Einwirkung der Rembrandtschen Schule. In goldbrauncm Ton flüchtig gemalt. Signirt: R. Troyen fec.

Pieter Claesz. Geräucherte Häringe. Signirt PC 1627. Noch ein drittes Bild ohne Signatur.

Jan Steen zugeschrieben. Tischgebet. Das Bild ist nicht von Jan Steen.

Aernout Smit. Zwei Marinen. Feiner, grauer Ton. Herrlicher, frischer Wellenschlag. A.SMIT signirt.

Christoffel van Der LAmen. Elegante Gesellschaft, die in einem Parke mit den Kolben spielt. Hohe, steife Figuren. Die Landschaft breit gemalt. Das Bild ist bezeichnet: $C_{H}$. VAN DER LAMEN I64I.

Ders. Elegante Gesellschaft in einem vornehmen Salon. Durchblick in einen anderen Salon. Mit der Jahreszahl I64I.

Ders. Herrschaftlicher Park mit vielen vornehmen Personen. Breit und flott gemalt. Es befinden sich in den Privat-Zimmern des Marchese MaNsI noch mehrere Bilder dieses sonst so seltenen Meisters. In der Galerie Consini zu Rom finden sich zwei, besonders in der Färbung sehr an RubENs erinnernde Gemälde. Die Bilder hier erinnern dagegen mehr an Anthonie Palamedesz.

Roelof Jansz. Van VRIEs. Sehr schöne an Ruisdaei, erinnernde Landschaft. Bezeichnet: RV R I ES.

Mathys Naiveu: Die Verkäuferin. In der Manier Dou's. Jedoch breiter als dieser und in kräftig leuchtenden Farben gemalt. Signirt: MN A I V E U I 669.

FrançoIS Snyders. Prachtvolles Fruchtstück. Die Figuren nicht von RUBENS wie angegeben ist.

Jan van Huchtenburgh. Zwei mächtige Schlachtenbilder.

Karel Dujardin. Schöne Landschaft von einem kleinen Strom durchrieselt, mit Hirtenstaffage. Wird Pynacker genannt, ist jedoch" echt bezeichnet: C. Dujardin. ${ }^{1}$ )

1) Nach einer früheren Notiz des Herrn Dr. Bredius. 



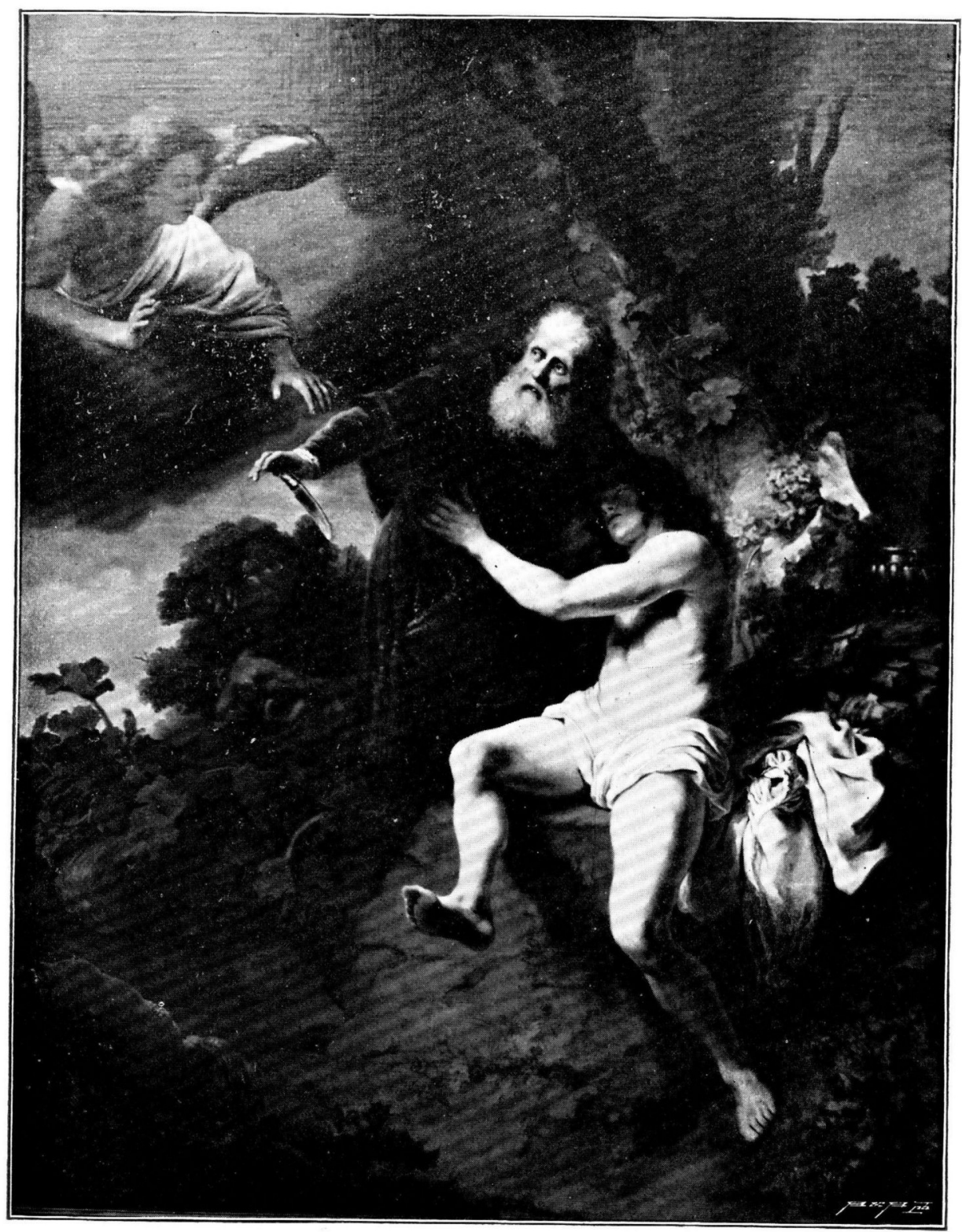

FERDINAND BOL.

Das Opfer A BRAHAms.

Galerie Mansi in Lueca. 


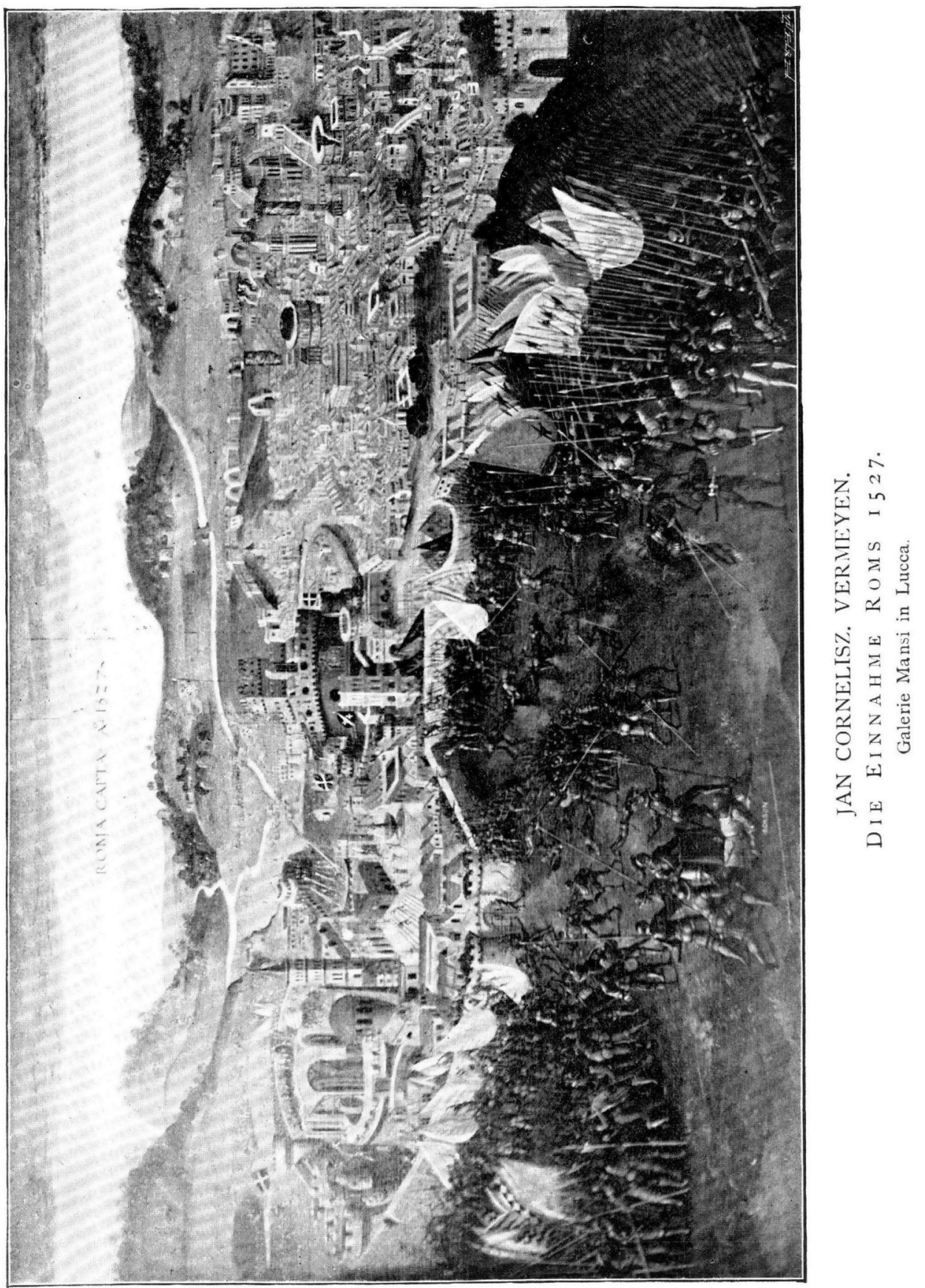



Jan Miense Molenaer. Baucrngelag.

Ferdinand Bol. Das Opfer Abrahams. Das kolossale Bild, welches noch der Frühzeit des Meisters angehört, ist vielleicht das bedeutendste seiner Werke ausserhalb Hollands. In den Figuren ein mächtiger Zug; dabei sind sie einfach, ohne Schwulst. Doch ist namentlich die Landschaft, besonders die Behandlung des Pflanzenwuchses, von erstaunlicher Grossartigkeit. Der dominirende Farbenton ist goldbraun, jedoch von einem bläulichen Schimmer leicht touchirt, der in den Schatten der Carnation stärker betont ist. Das Bild ist signirt: F. BoL fecit.

JaCob Ochtervelt. Ein Soldat in Besuch bei einer Dame. Ein farbenschönes Bild dieses feinen Rotterdamer Meisters. Bezeichnet: J. O. V.

Jan Cornelisz. Vermeyen. Sehr fein ausgeführtes Prospect oder Vogelperspektiv der Schlacht bei Tunis 1535. "Taunetum expugnatum A०. I535".

Derselbe. Die Einnahme Roms I 527 mit Prospert der Stadt um diese Zeit, af welchem man die Erbauung der Peterskirche gewahr wird „Roma Capta. $A^{\circ}$. 1527 ".

Derselbe. Ein drittes Bild, die Schlacht bei Pavia 1525. "Captio Regis Fra: Ao. 1525". In ähnlicher Weise behandelt. Die drei Bilder sind wahrscheinlich als Vorlagen für Teppichwirkerei zu betrachten. Einige ähnrliche Darstellungen im Schloss zu Coburg. Sonst ist der Meister selten geworden. Wenn ich die Vorlagen zu den in Madrid sich befindenden Teppichen mit Darstellungen aus den Feldzügen KARL V. in Tunis, (jetzt in der kaiserlichen Galerie zu Wien ausgestellt, früher unzugänglich), ausnehme, weiss ich nichts mehr, was man dem seiner Zeit so berühmten Meister zuschreiben kann.

P. Schormis (?) Herrlich leuchtendes Stilleben mit todten Vögeln und hängenden Hasen, in grüngelbem, metallenem Ton gemalt. Bezeichnet: P. Schormis (?) (Vielleicht Pouwels Schotanus ?)

Gyskert d'Hondecoeter. Hühnerhof mit einem Pfau. Von bedeutender koloristischer Feinheit. Bezeichnet: G D H (verschlungen) I632.

Wüstenlandschaft mit Staffage. Nicht von REMBrandT, wie angegeben wird. Eher dem BARTHOLOMEUS BREENBERG ähnlich, jedoch schwächer.

Die Versuchungen des hl. Antonius. Von HERRI BLes oder von einem verwanditen Meister. 
Johannes Lingeleach. Grosses Hafenbild. Voll bezeichnet: Johan LINGELBACH me fecit.

Adam Willaerts. Zwei Marinebilder. Graugelber Ton. Beide bezeichnet: $A, W .1640$.

JaN VAN GOyen. Zwei Marinen. Namentlich das eine Bild, mit einer Burg am Gestade, sehr schön.

Ludolf Bakfuijsen. Grosse Marine. Bezeichnet: $\mathrm{BH}$

Fruchtstück (Äpfel) im goldenen Ton gemalt. Soll CJ VAN KEVLEN bezeichnet sein.

Lardschaft mit Diana im Bade. Staffage. In der Manier Cuylenborchs. Bezeichnet: Po. G. V.

Rombout van Troyen. Noch zwei Bilder dieses sonst ziemlich seltenen Meisters. Sie stellen einen geheimnisvollen Vorgang in einer Grotte dar. Goldbraun leuchtend in der Farbe. Flüchtige, prickende Pinselführung.

Verschiedene Stilleben werden Pieter de Witte (Pittro Candido) zugeschrieben, sind aber von Pieter Clatesz.

Daniel Seghers. Maria mit dem Kinde als Basrelief dargestellt, en grisaille gemalt, aber von einem reich gefärbten Blumenkranz umgeben.

David Teniers Jr. Der verlorene Sohn. Das Bild ist nur eine alte Copie, und zwar, wenn ich mich nicht täusche, von dem schönen Bilde desselben Gegenstandes in der Berliner Gallerie.

Pie ter Brueghel Jr. Vier grosse Bilder, ländliche Feste und Ähnliches darstellend. Alle bezeichnet I'. BRUEGHEL 1620. Es geht schon aus dem Datum hervor, dass nicht der ältere, sondern der jüngere PIETER BRuEGhel der Meister ist. Die kräftig gefärbten lebendigen Schilderungen stehen jedoch, was Feinheit und Charakteristik anbetrifft, hinter den Bildern des Vaters beträchtlich zurück. (Beschrieben von BREDIUs im Nederl. Kunstbode.)

Der heilige Hieronymus in seiner Zelle. Sehr gutes Bild in der Art des Marinus VAN REyMerswale. 
Nicolaes Berchem. Nachtstück. Fackeltragende Hirten ihr Vieh treibend. Schwacher Mondschein. Feine, für Berchem ungewöhnliche Beleuchtung. Undeutlich bezeichnet: BERGHEM 1652 (?)

Lucas Van Leyden zugeschrieben. Anbetung der Könige. Das etwas roh ausgeführte, jedoch charaktervolle und fliessend gemalte Bild ist nicht von LUCAS VAN LEYDEN, (wie häufig wird dieser Name nicht missbraucht!) sondern von einem mir unbekannten Niederländer. Es ist bezeichnet $£$ I523 (ob echt?)

Melchior D'Hondecoeter. Zwei prachtvolle grosse Hühnerhofbilder. Bezeichnet.

GERARD TER, BorCH zugeschrieben. Zwei Bildnisse. Mann und Frau. Lebensgrosse Kniestücke. Sehr tüchtige Portraits, aber nicht von TER BORCH.

Dirck van Babueren. Christus im Tempel. Datirtes Bild dieses seltenen Utrechter Meisters.

DE Weth. Z Zei.Bilder, mit Darstellungen von Priestern.

PAUlus Brill. Landschaft.

Thomas Wyck. Zwei bezeichnete Bilder.

Die Galerie GIAN BATt. MANSI (Piazza Maria Bianca).

1. Saal Nr. 5. Quentin Massys zugeschrieben. "Quadro allegorico". Das Bild stellte eine Art Memento Mori dar. Eine junge Laute spielende Frau, welcher ein älterer Mann zwei Todtenköpfe präsentirt. Mit dem Spruche: "Het is beter te sterve dan in wellust te leve". Das Bild ist bei weitem nicht fein genug für Quentin Massys, wenn es auch an seine Manier erinnert.

N.. I I. LuCas Van Leyden zugeschrieben. Die hl. Magdalena. Sie ist mit einem Mantel von braunrothem Sammt prachtvoll bekleidet. Auf dem Kopf trägt sie eine Haube mit feiner Goldstickerei und einem Edelsteingeschmeide geziert. Hinter ihr zeigt sich eine herrliche Felsenlandschaft, weiter zurück gegen den Horizont werden ein blaugrüner See und niedrige Berge sichtbar. Dieses schöne Bild, das mit LUCAS von LEyden nichts zu thun hat, dürfte dagegen wirklich von QUENTIN Massys herrühren. ${ }^{1}$ ) Der

1) Auch dar "Cicerone" schreibt das Bild dem Quentin Massys zu.

Oud-Holland, I896. 
Typus des länglichen, fein geschnittenen Geșichtes, die bleiche Carnation mit den schwachen, olivenartigen Schatten, deuten auf ihn hin. Das in herrlichen Farben wunderbar leuchtende Bild kann zu den schönstcn der in Italien sich befindenden altniederländischen Gemälden gerechnet werden.

2. Saal Nr. 24. P. P. Rubens zugeschrieben. Martyrium eines heiligen Bischofs. Skizze. Nicht von RUBENs, sondern nur eine schwache Nachahmung oder Copie.

3. Saal Nr. I3. P. P. Rubens zugeschrieben. Die mystische Vermählung der h1. Catharina im Beisein mehrerer Heiligen. Die Farben sind prächtig genug, die Typen aber flau. Von einem Nachahmer des RuBENS.

4. Saal Nr. I4. Maria, die dem nackten Christkind ein Glas Wein zu trinken gibt. Ein feines Bildchen des Meisters vom Tode der Maria.

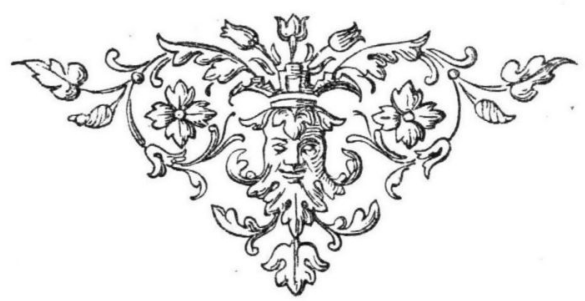

